\title{
CAF Implementation on FPGA Using Python Tools
}

\author{
Chiranth Siddappa $a^{*}$, Mark Wickert
}

\begin{abstract}
The purpose of this project is to provide a real time geolocation solution by generating code for the complex ambiguity function (CAF) in a hardware description language (HDL) and the implementation on FPGA hardware. The CAF has many practical applications, the more traditional being radar or sonar type systems. By using scientific Python tools, this project provides a solution for testing signals and the ability to customize modules to target multiple devices. The processing for this implementation will be done on a PYNQ board designed by Xilinx. The PYNQ board provides a Zynq chip which has both an ARM CPU and FPGA fabric. All required mathematical operations for the CAF are returned to the user through Python classes which produce synthesizable code in the Verilog HDL. The Python classes use Jinja templates integrated into the Verilog code to allow for configuration changes that a user will need to change for investigation and simulation, development, and test. Helper methods are included in the package to help simulation of the HDL such as quantization, complex data reading and writing, and methods to verify the data using quantized values.
\end{abstract}

Index Terms—complex, ambiguity, function, overlay, verilog, jinja, jupyter, xilinx, fpga, zynq, pynq, linux

\section{Introduction}

In this investigation, the pre-processing steps of downsampling and filtering are simulated and considered outside of the scope of this project. In the case of geolocation systems, the use of collectors and reference emitters are used to create geometries that will allow for the detection of Doppler and movement in the signal. The Doppler is used to calculate a frequency difference of arrival (FDOA). Then, cross correlations can be used to determine the time delay by denoting the peak location of the resulting output as a time delay of arrival (TDOA). The goal of this project is to be able to provide a real time solution for FDOA and TDOA. The basic algorithm for calculating the complex ambiguity function for time difference of arrival and frequency offset (CAF) has been well known since the early 1980's [Ste81]. In many radio frequency applications, there is a need to find a time lag of the signal or the frequency offset of a signal. The reader would be familiar with a form of frequency offset known as Doppler as a common example. The CAF is the joint time offset and frequency offset generalization. The CAF was mainly used first for radar and sonar type processing for locating objects using a method known as active echo location [KPK81]. In this scenario, a matched filter design would be used to ensure that the signals match [Wei94]. More commonly with newer radio frequency systems such as

* Corresponding author: csiddapp@uccs.edu

₹University of Colorado Colorado Springs

Copyright $\odot 2019$ Chiranth Siddappa et al. This is an open-access article distributed under the terms of the Creative Commons Attribution License, which permits unrestricted use, distribution, and reproduction in any medium, provided the original author and source are credited.
GPS, similar but orthogonal signals are transmitted in the same frequency range. Because of the property of orthogonal signals not cross correlating they do not collide with one another, and they are an optimal signal type for testing this application [ZT08].

\section{Motivation}

The CAF has many practical applications, the more traditional being the aforementioned radar and sonar type systems, with a similar use case in image processing. The use of cross-correlations in the form of the dot product to find similarities is the same theoretical basis for our use in geolocation. In the particular case of geolocation systems, the use of collectors and reference emitters are used to create geometries that will allow for the detection of Doppler and movement in the signal. This method of calculation has yet to be simplified. Currently GPU's have been employed as the main workhorse due to the availability as a co-prorcessor. But the use of the FPGA has always been an attractive alternative due to the high configurability of the hardware options, but comes with much higher up front design cost [HP17]. For design cost, we are primarily concerned with the development time for code that can be written in C syntax in the form of OpenCL or CUDA for a GPU, as compared to using an HDL which will require background in digital logic and testing that must occur on hardware directly.

To geolocate a signal emitter's location the Doppler is used to calculate a frequency difference of arrival (FDOA) which represents a satellite's drift. Then, cross correlations can be used to determine the time delay by denoting the peak of the resulting output as a time delay of arrival (TDOA). The refernce signal will be different for every use case, which motivates the need to ensure that the resulting Verilog hardware description language (HDL) module output can also be produced to match necessary configurations [ver01]. This became a project goal motivated off work done by other projects to be able to produce code in other languages [Sym]. Thus, the solution provided must be able to be reconfigured based off of different needs. The processing for this system will be targeted to a PYNQ board manufactured by Xilinx, but has been designed such that it can be synthesized to any target device. All Verilog HDL modules that are produced by the Python classes conform to the AXI bus standards of interfacing [Arm17]. This allows for a streamlined plug and play connection between all the modules and is the basis of the templating that is implemented with the help of Jinja.

\section{Starting Point}

The main concepts necessary for the understanding of the CAF are topics that are covered in Modern Digital Signal Processing, Communication Systems, and a digital design course. These concepts 


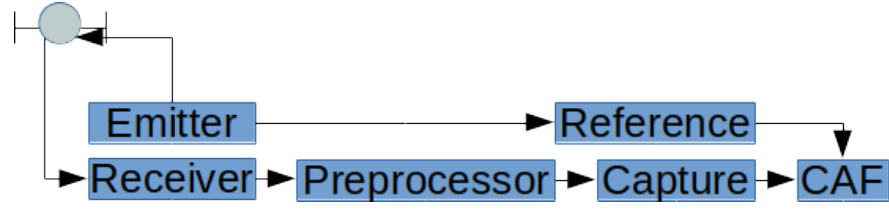

Fig. 1: Satellite Block Diagram for Emitter and Receiver.

would be the Fast Fourier Transform (FFT), integration in both infinite and discrete forms, frequency shifting, and digital design. This project will show a working implementation of digital design HDL modules implementing the logic accurately with this given knowledge. Given the mathematical background of this project, it is crucial to have a way to test implementations against theory. This is the motivation for the discussion of using Python to help generate code and test benches.

\section{Project Overview}

The goal of this project was to implement the CAF in an HDL such that the end product can be targeted to any device. The execution of this goal was taken as a bottom up design approach, and as such the discussion starts from small elements to larger ones. The steps taken were in the following order:

1) Obtain and generate a working CAF simulation

2) Break simulation into workable modules

3) Design modules

4) Verify and generate with test benches

5) Assemble larger modules

6) Synthesize and Implement using Vivado for the PYNQ$\mathrm{Z} 1$ board

\section{Complex Ambiguity Function}

An example of the signal path in the satellite receiver scenario is described by Fig. 1. In this case, an emitted signal is sent to a satellite, and then received and captured by an RF receiver. Some amount of offset is expected to have happened during the physical relay of the signal back to a receiver within the broadcast area of the satellite. The signal is then downconverted and filtered, and then sent to the CAF via a capture buffer. While a signal is sent through an upconverter and relayed to the satellite, a copy of the same signal must be stored away as a reference to compute the TDOA and FDOA. Both the reference and capture blocks are abstractions, and have individual modules written in Verilog to handle the storage of these signals.

Another very specific example of the satellite receiver scenario is described by Fig. 2. In this scenario, we see that no emitter exists, yet a reference signal is able to be sent to the CAF for TDOA and FDOA calculations. This is because GPS signals use a PRN sequence as ranging codes, and the taps for the signals are provided to the user [ $\mathrm{Na18}$ ]. This provides a significant processing gain as the expected sequence can be computed in real time or stored locally. This project takes advantage of these signals through the use of gps-helper [WSa].

As a basis for what the rest of this paper is describing, an overview of the CAF and the various forms of computing are provided.

The general form of the $\mathrm{CAF}$ is:

$$
\chi(\tau, f)=\int_{-\infty}^{\infty} s(t) s^{*}(t-\tau) e^{i 2 \pi\left(f / f_{s}\right) t} d t, \frac{-f_{s}}{2}<f<\frac{f_{s}}{2}
$$

The equation describes both a time offset $\tau$ and a frequency offset $f$ that are used to create a surface. The frequency shift $f$ is bounded by half the sampling rate. The discrete form is a little simpler, and lends itself to the direct implementation [Har05]:

$$
\chi(k, f)=\sum_{n=0}^{N-1} s[n] s^{*}[n-k] e^{i 2 \pi\left(f / f_{s}\right)(n / N)}, \frac{-f_{s}}{2}<f<\frac{f_{s}}{2}
$$

where $N$ is the signal capture window length, $f_{s}$ is the sampling rate in $\mathrm{Hz}$ making $f$ have units of $\mathrm{Hz}$ and $k D$ is a discrete time offset in samples with sample period $1 / f_{s}$. In both the continuous and discrete-time domains, $\chi$ is a function of both time offset and frequency offset. The symbol $s$ represents the signal in question, generally considered to be the reference signal. The accompanying $s^{*}$ is the complex conjugate and time shifted signal.

As an example, a signal that was not time shifted would simply be the autocorrelation [ZT08]. It is referred to as the received signal in this context, and it is the signal that is used to determine both the time and frequency offset. To determine this offset, we are attempting to shift the signal as close as possible to the original reference signal. The time offset is what allows for the computation of a TDOA, and the frequency offset is what allows for the computation of the FDOA.

In this implementation, the frequency offset is created by a signal generator and a complex multiply module that are both configurable. Once this offset has been applied, a cross-correlation is applied directly in the form of the dot product. This eliminates the costly implementation case where an FFT and an inverse FFT are used to produce a result. The signal generator can supply a specified frequency step and accuracy with configuration of the signal generator class [Sida]. An example of the signal generator is shown in Fig. 9. The resulting spectrum is shown in Fig. 8. This satisfies the frequency $(f)$ portion of the equation. The complex multiply module is similarly configurable for different bit widths through the complex multiply generator class [Sida]. An example CAF surface is provided in Fig. 3 showing how the energy of the signal is spread over both frequency and time. This type of visualization is very useful for real-world signals with associated noise. In this project, care was taken in truncation choices to ensure that the correlation summation ensures signal energy retention. In this project, the CAF module that has been implemented will return a time offset index and frequency offset index back to the user based off provided build parameters shown in the code listing for the Python class $C A F$, described in a later section for the CAF Module. When writing the module, all simulation and testing was done at the sample by sample level to ensure validity so the CAF surface was not used in testing. A method for computing the CAF using the dot product and frequency shifts has been published to the package. This implementation is specific to this project in that it uses a sample size that is twice that of the reference signal for the computation. A sample output slice will be shown in the Experiments section for the CAF module in Fig. 16. 


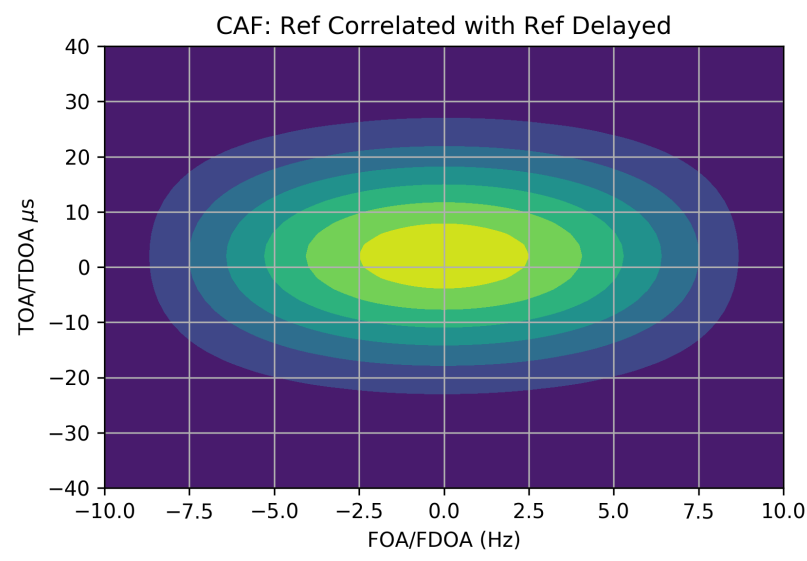

Fig. 3: CAF Surface Example.

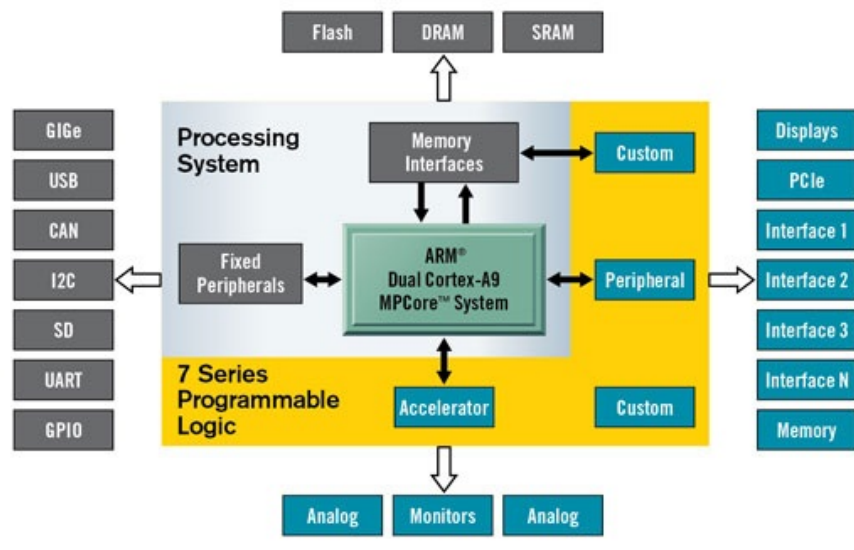

Fig. 4: The $P Y N Q$ processing overlay diagram. [Xilb]

\section{Hardware}

The targeted hardware for this project is the Zynq processor on the PYNQ-Z1 board. However, this project is fully synthesizable and should be able to be targeted for any other Xilinx board.

\section{Python and PYNQ}

The PYNQ development board designed by Xilinx provides a Zynq chip which has an ARM CPU running at $650 \mathrm{MHz}$ and an FPGA fabric that is programmable via an overlay [Xilb]. This performance allows for a linux operating system to be run on the CPU which in this case is Ubuntu, and hosts a Jupyter notebook to program and interface with the FPGA fabric using an overlay. This overlay contains mappings for ports and interfaces between the fabric and the CPU. This functionality is very unique in that both an ARM core and a fabric are on the same board. As shown by Fig. 4 the overlay sits between the processing system (CPU) and the programmable logic (FPGA). This overlay is loaded and programmed to the fabric through a Jupyter notebook and allows for native visualization and data interaction through any Python tools that work inside the IPython kernel. The overlay is represented by the yellow background with labels "Custom" and "Accelerator" and shows how the overlay is a communication layer between the processing system and the programmable logic.

It also contains a bitfile that will properly configure the FPGA [Xilc]. This bitfile is generated through the Vivado Design Suite that is provided by Xilinx by loading the output modules from the

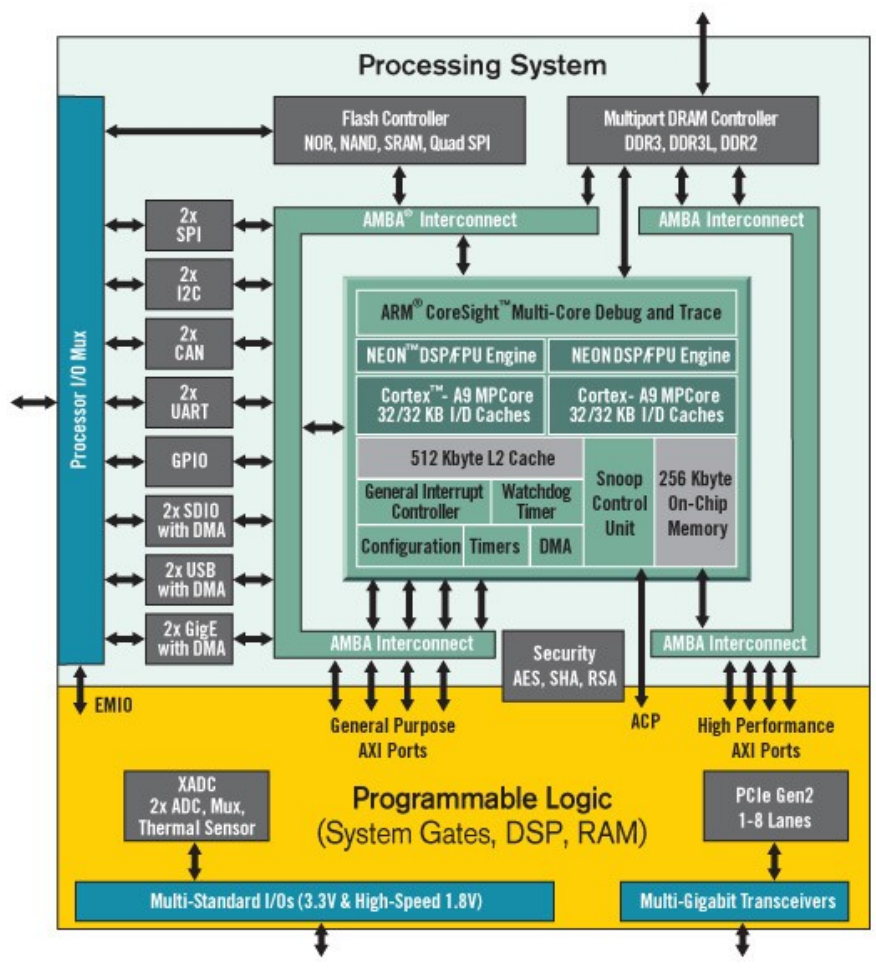

Fig. 5: The PYNQ processing overlay diagram. [Xilb]

caf-verilog module. A different bitfile must be created for every unique combination of configuration of the CAF and every device that is targeted. Every instantiation of the CAF Python class that has different parameters will require a new bitfile.

The Jupyter notebook itself is considered an interactive computing pool providing an interface to do computation and prototyping through a web browser. In this implementation it is meant to be an easier way for a non-hardware oriented person to be able to access a computational accelerator designed by a hardware engineer [Xilb].

A diagram of the processing and the programmable logic is shown in Fig. 5. The processor system is the Cortex-A9 processor that is running at $650 \mathrm{MHz}$ with $512 \mathrm{MB}$ of DDR3 RAM. The FPGA is a Zynq XC7020 part which has 13,300 logic slices, 53,200 6-input LUTs, 160,400 flip-flops, 630KB of block RAM, and 220 DSP slices. Later, a usage report is provided with a description of how the logic was optimized to make use of these primitives. It is possible to access the DRAM from the programmable logic (FPGA) through an AXI IP Core.

\section{Software}

Xilinx Vivado WebPack 2018.2

The Vivado design tool provides a simulator along with the ability to synthesize, elaborate, and implement the design [Xil14]. For this project, this built-in simulator was used exclusively. Other simulators were not chosen because the other target devices that this project seeks to be implemented on are likely to also be Xilinx products. The tool is free to download for anyone to use, and allows the hardware engineer to develop and synthesize HDL designs for Xilinx FPGA's. There is also a Software Development Kit that allows an engineer to write in $\mathrm{C}$ code. For this project, all modules are written in Verilog. This was done because of the need to instantiate multiple submodules that provide functionality 
together. When running the synthesis tool, the output was very useful in helping make incremental design changes to fully optimize the board. Although none were used in this project, Xilinx does offer many free IP Cores that can be used in designs. They are black boxes that can be used in both simulation and the final implementation in HDL and block designs.

\section{Python and Jupyter}

This project made extensive use of the Python ecosystem through the use of pip, Jupyter, and many other packages. The reader is encouraged to view the caf-verilog source code [Sida] and view the releases that have been made on PyPI [Sidb]. When designing modules, a first test of what a signal should look like when operated on was done using the interactive plotting ability that is provided [Pro]. The generation of the modules was done using Jinja which provides both template parsing and rendering [Ron]. Whenever a simulated signal was changed, instead of having to write out a file or test bench by hand, a template was used to create the output and render it to the simulation directory. The signals that are used to create the signal generator were first quantized by using the NumPy library and then written to a file that gets used a memory buffer in the signal generator [Num]. Most of the mathematical operations that are implemented were first verified using this library. This project requires the use of orthogonal signals to ensure that the spectral density that is being tested is isolated from the others. This was possible using the gps-helper module that implements the GPS gold codes that are orthogonal PRN sequences [WSa].

\section{Quantization}

In order to use a signal in the digital domain, a signal must first be quantized by an analog to digital converter (ADC). Most ADC's that are available are able to provide a 12-bit value, and some newer devices are now able to provide 16-bits [Ana]. However, for this project 12-bit signed signals were used during testing as this is a very nice number to compute mentally and still provides minimal energy loss when plotting on the spectrum.

Inspecting signals after quantization is important because when signals are reduced in size there is information loss. This is demonstrated by Fig. 6 where a 12 bit and 8 bit quantization of a cosine signal is shown. Quantization helper functions are provided in caf_verilog with the help of scikit-dsp-comm's simpleQuant function [WSb]. This means that the full bit value of the signal cannot be used otherwise there is signal loss to DC gain. The signals must be equal over 0 . For a 12-bit quantization of a vector for example the numbers must be in the range $(-4095,4095)$ in comparison to the two's complement full value of $(-4096,4095)$. This is all necessary because the computation that is done on the FPGA will be done using fixed point or an integer value. This also reduces power and cost on the FPGA [FR]. Test files are written out and read back as integer values via this module by all the other classes for tests and verification.

\section{Complex Multiply}

As an example for why this module is necessary, an example of frequency shifting a signal is presented. In Fig. 7 we have two inputs: a positive frequency signal on top, and a negative frequency signal in the middle. The output is shown in the bottom plot. All of these signals are shown in a spectral density plot, with both sampling frequencies normalized to a value of 1 for presentation. What we see is that the resulting spectrum has a

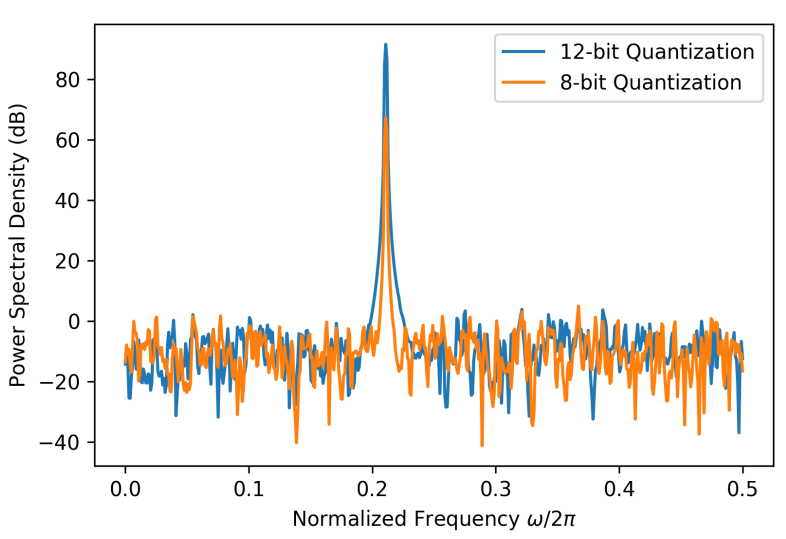

Fig. 6: 12-bit and 8-bit Quantization Comparison.

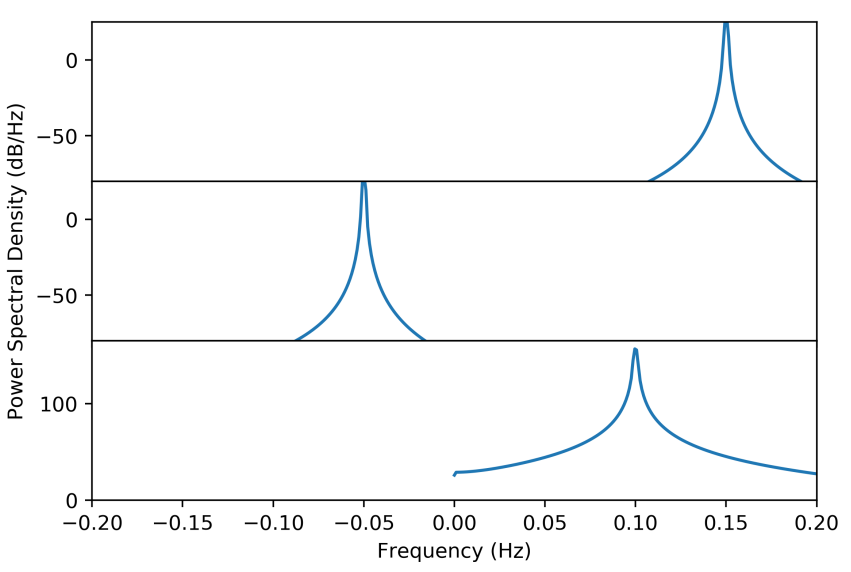

Fig. 7: Inputs (top and middle) and output (bottom) of the CPX Multiply Verilog module.

signal at a frequency of the sum of the two negative and positive frequency signals. This is what is expected. This method is what is used to shift the captured signal for the CAF.

Signed multiplication in Verilog can be done by specifying the signed data type. Any multiply of two numbers of the same size requires twice the number of bits in the result [Tum]. However, in this project the need for different size operands arises. This module takes in two complex numbers and performs a pipelined multiplication on the data. Before the result is provided to the master, the result is truncated. It should be noted that no timing constraint violations were encountered during the implementation. The only timing constraint that was provided was the slew rate for the fabric clock, and all other constraints were Vivado defaults.

The specific pipeline steps are presented in Table 1 which shows which operations are completed in which pipeline stage. Stages 1 and 2 are always conditionally assigned based on the current state of the AXI interface so that resources are not constantly being used. This helps for timing and for power usage. The result is then truncated and returned to the master when the master's ready signal is asserted. Because this is a pipelined implementation, an input and output can be processed every clock.

A code listing of the Verilog HDL output is provided as reference. The two blocks that are shown are for the first step 
TABLE 1: CPX Multiply Stages

\begin{tabular}{ll}
\hline Stage & Operation \\
\hline 1 & $\mathrm{xi} * \mathrm{yi}$ \\
1 & $\mathrm{xq} * \mathrm{yq}$ \\
1 & $\mathrm{xi} * \mathrm{yq}$ \\
1 & $\mathrm{xq} * \mathrm{yi}$ \\
2 & $\mathrm{xu}-\mathrm{yv}$ \\
2 & $\mathrm{xv}+\mathrm{yu}$ \\
3 & Truncate
\end{tabular}

through the third step. The first two steps can be seen to only be calculated when the master signal conditions are correct.

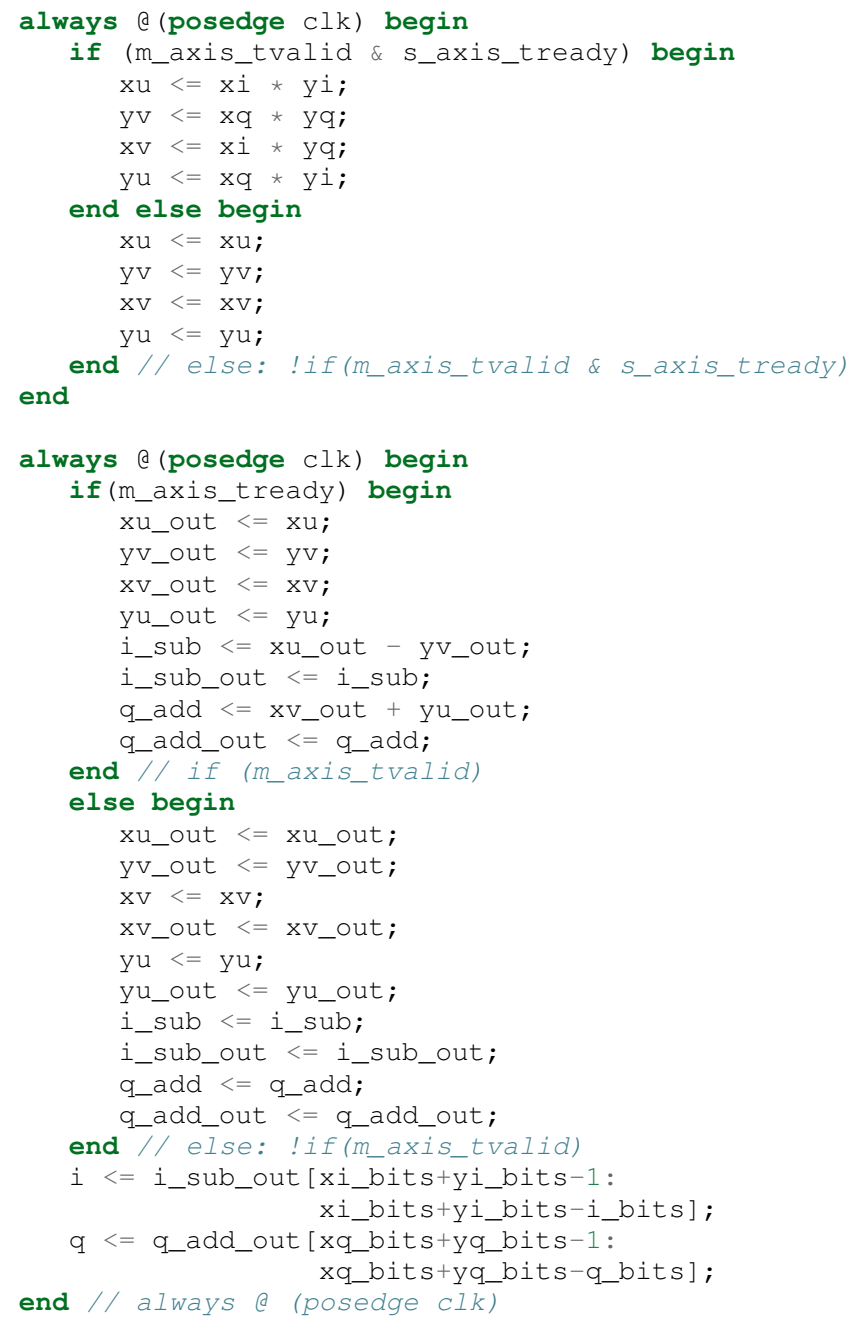

\section{Signal Generator}

The signal generator module is implemented using a half sine lookup table and accumulator. This is commonly known as a numerically controlled oscillator in direct digital synthesis [MS]. This module produces a sine wave at the specified frequency by using a modulo counter that increments a phase value at every clock cycle. Note that the sampling frequency of the signal, $625 \mathrm{kHz}$, is different from the clock frequency of the board, at $250 \mathrm{MHz}$. The number of phase bits that are necessary are determined by the sampling frequency and the frequency resolution specified by Eq. 1.

$$
\left\lceil\log _{2}\left(\frac{f_{\text {clk }}}{\text { freq_res }}\right)\right\rceil=\text { phase_bits }
$$

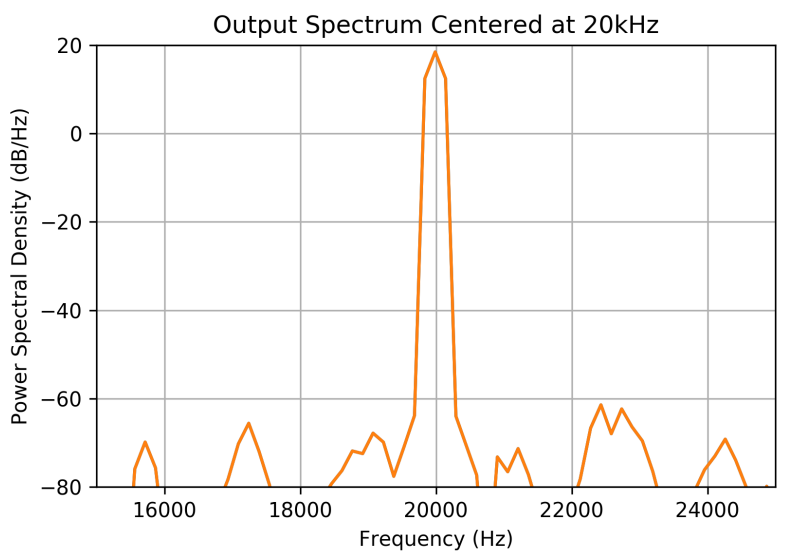

Fig. 8: Cosine centered at $20 \mathrm{kHz}$ with 8-bits and $200 \mathrm{~Hz}$ resolution.

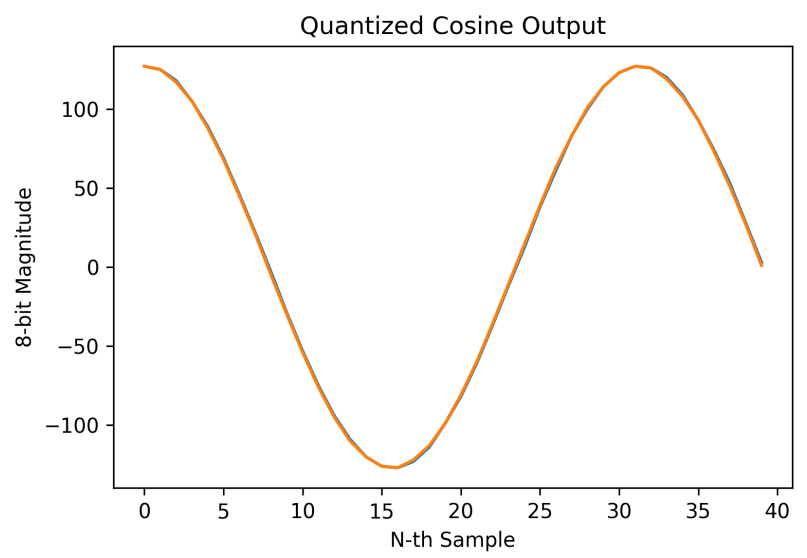

Fig. 9: Cosine centered at $20 \mathrm{kHz}$ with 8-bits and $100 \mathrm{~Hz}$ resolution.

The output of one cycle is shown in Fig. 9. The values that are supplied to the module for the lookup table are generated using the NumPy sine function and are quantized using helper methods included in the caf_verilog module. To set the frequency of the signal generator, a phase step or increment value must be provided by Eq. 2 .

$$
\frac{f_{\text {out }} \cdot 2^{\text {phase_bits }}}{f_{\text {clk }}}=\text { phase_increment }
$$

An example spectrum of the output of the signal generator that is created from the Python class is shown in Fig. 8. While no calculation of power has been provided, a parameter n_bits sets the signal strength. For this project, a value of 8-bits was found to be sufficient to provide a frequency shifted signal. The same settings used to generate the module used as an example in this section are used in Fig. 10 by using the SigGen class.

\section{Frequency Shift}

The frequency shift module takes in the same parameters as the signal generator module and adds an input for a complex value to shift. This module needs to make sure that different bit width signals are multiplied together correctly and that the pipeline is managed correctly to ensure that there are no phase shifts. Fig. 10 shows an input signal, and the resulting shifted signal. When using the Python generated Verilog module, a negative value for the frequency will be taken care of by setting a bit in the 


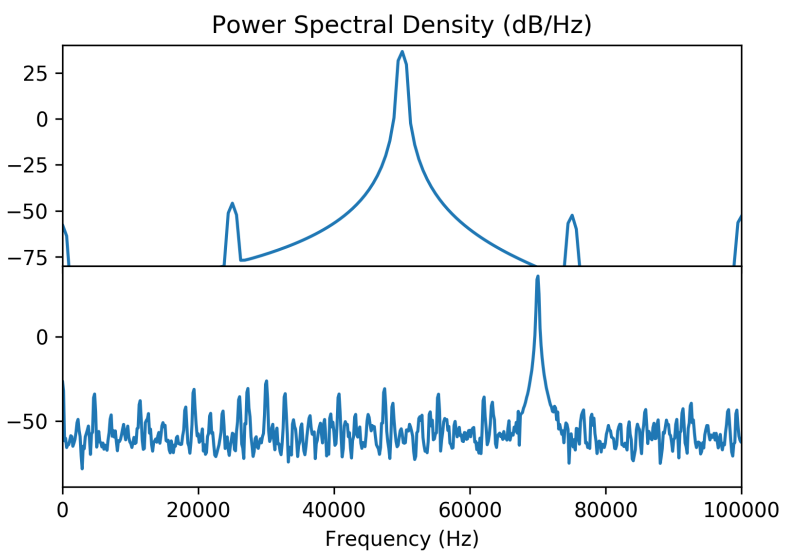

Fig. 10: Input signal at $50 \mathrm{kHz}$ (top), and output signal at $70 \mathrm{kHz}$ (bottom).

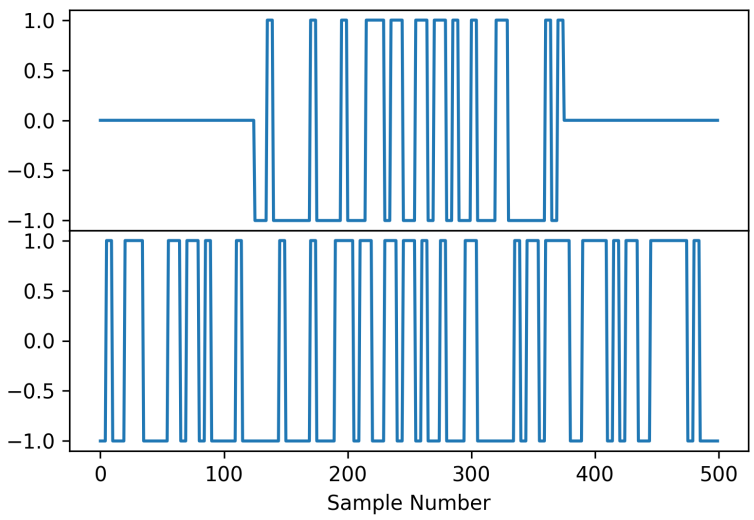

Fig. 11: A reference and received signal (top and bottom) simulated from a PRN sequence from gps-helper.

module parameters to perform the complex conjugate on the signal generator output.

Required inputs for the FreqShift Python class are the input vector ' $\mathrm{x}$ ', and the number of bits for the $\mathrm{I}$ and $\mathrm{Q}$ data that it represents. The same parameters are passed to the FreqShift class so that the SigGen module can be instantiated internally and accessed for naming by the Jinja template for the module.

\section{Cross Correlation}

The cross correlation is useful in comparing the time offset between two signals. As an example, a pseudorandom sequence signal provided by gps-helper [WSa] is time shifted in Fig. 11 by ten samples. Both of these signals are a non-return to zero representation of the binary bit sequence. The reference is shown with zero padding on either end so the visual representation stays centered between the two signals.

The general form of the cross correlation is [ZT08]:

$$
(f \star g)(\tau)=\int_{-\infty}^{\infty} \overline{f(t)} g(t+\tau) d t
$$

In Eq. 3 the signal $f(t)$ is shown with the complex conjugate, and the signal $g(t)$ is shown with a time or sample shift of $\tau$. When

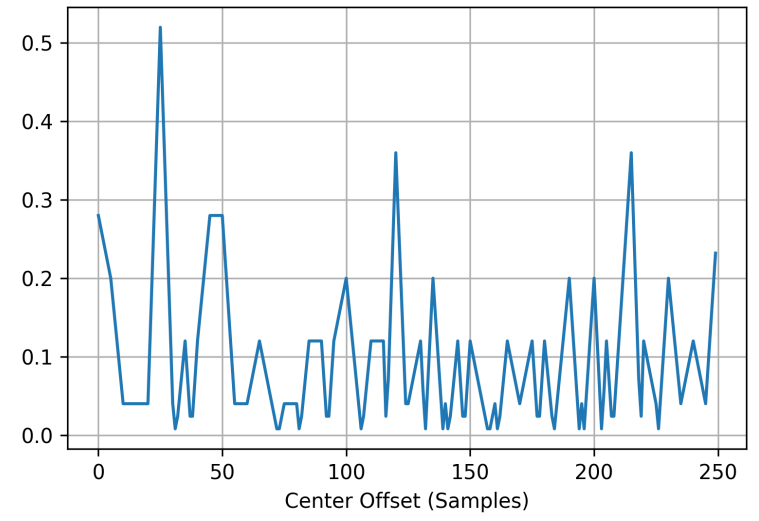

Fig. 12: The cross correlation of two simulated signals, showing a positive offset of 25 samples using xcorr.
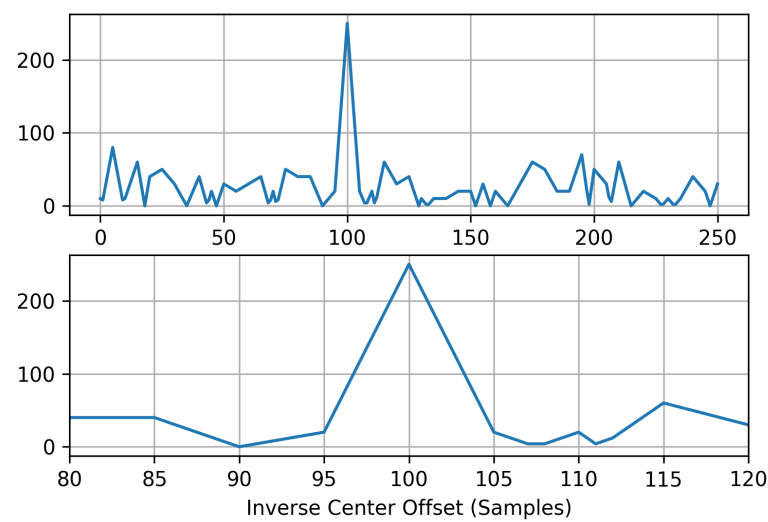

Fig. 13: Cross correlated output of a signal of length 250.

translated into the discrete form, the form looks like the following:

$$
(f \star g)[n]=\sum_{m=-\infty}^{\infty} f^{*}[m] g[m+n]
$$

When looking at the cross product in discrete form (4), it is possible to see that the form of the multiplication and addition closely follows that of the dot product (Eq. 5).

$$
a \cdot b=\sum_{i=1}^{n} a_{i} * b_{i}
$$

These series of equations provide a means of determining where the signals are correlated in time, or if they are orthogonal meaning they are not correlated at all [ZT08]. In order to capture the full signal power with the dot product, it is necessary to store twice the length of the reference signal for correlation as shown in Fig. 11. Furthermore, as compared to Fig. 12 which uses xcorr, the dot product method that produced Fig. 13 has a much higher magnitude. This is because the xcorr method uses an FFT, and the result is normalized to one. We also note that the axis for samples is denoted as an inverse offset. When the peak is generated with dot products, the center is going to be the center of the sample length. This is because the multiply and accumulate has the highest magnitude in the center as compared to the xcorr method with FFT's which produces a normalized axis around zero. Since we are always looking at what offset is necessary to cause the shift 
back to the reference, it is left as a sample offset. This also makes verifying the Verilog test benches much more straight forward. However, with the dot product, the magnitude that is achieved when a full correlation is hit is the length of the correlation sequence itself. This means that a longer integration time allows for a higher fidelity difference between signal magnitudes of surrounding shifted correlations. This method reduces the amount of multiplies that are necessary and is much simpler to implement on an FPGA. These results were verified using the xcorr function from scikit-dsp-comm and the dot product function provided by NumPy. The simulation for this function required the output of the entire sequence to be written to and sequentially read from disk. When running the simulations it was found that the very last dot product in the sequence was missing. A full cross correlation using the dot product actually has two times the length plus one to account for both positive and negative offsets.

\section{Dot Product}

The final CAF solution uses a pipelined multiply and accumulate. When the implementation was run, it was found that a pipelined implementation was able to make use of the primitive DSP48 type. Further fine-tuning suggestions were taken to ensure that the multiply and accumulate functionality of the primitive type was taken advantage of correctly [FWS].

\section{ArgMax}

Because there is a need to compare the magnitudes of complex numbers, the argmax function is required. The mathematical absolute value of a complex number is described in Eq. 6. However, finding the true absolute value of the number requires the implementation of the square root. The first option that was looked at was a binary square root algorithm [Min13] that only uses base 2 division. However, this can take a variable amount of clock cycles. An implementation is provided in the sqrt package as reference. The other option is the CORDIC logic core provided by Xilinx which also would apply backpressure [Xila], essentially sequentially buffering the result by a fixed number of clocks.

$$
r=\sqrt{x^{2}+y^{2}}
$$

After comparing results and performing the argmax using these different methods a decision was made to just use the squares of each of the real and imaginary components. This is possible because we can use the proportion of the squared values and their square roots to compute the argmax with the same result. Since the largest magnitude squared value is made up of both a real and imaginary component, it is enough to say that the largest magnitude $\left(x^{2}+y^{2}\right)$ will be sufficient. The result is provided back by the next clock, with only a delay in the pipeline for the first multiply. Then, comparisons are done within the module itself to find the max. This also allows for taking advantage of the larger integration time by allowing larger max values to propagate through. The trade-off is that there is much larger utilization with multiple instantiations all growing in size as the multiply operands increase in size. Inspecting the utilization of the synthesized and implemented designs did not seem to indicate that this was the limiting factor in the design layout growth.

\section{CAF Module}

The CAF module uses a generate variable, which is part of the Verilog standard [ver01] to implement the frequency shifts and

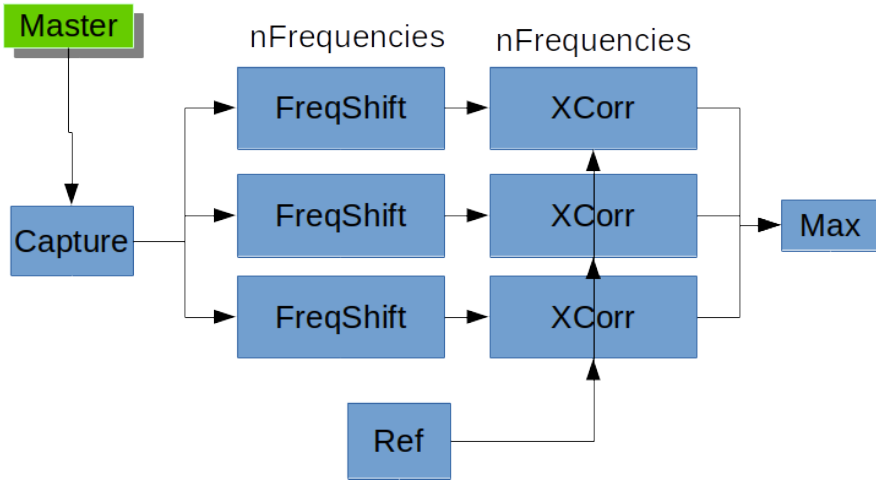

Fig. 14: Block diagram of a CAF implementation with three frequencies.

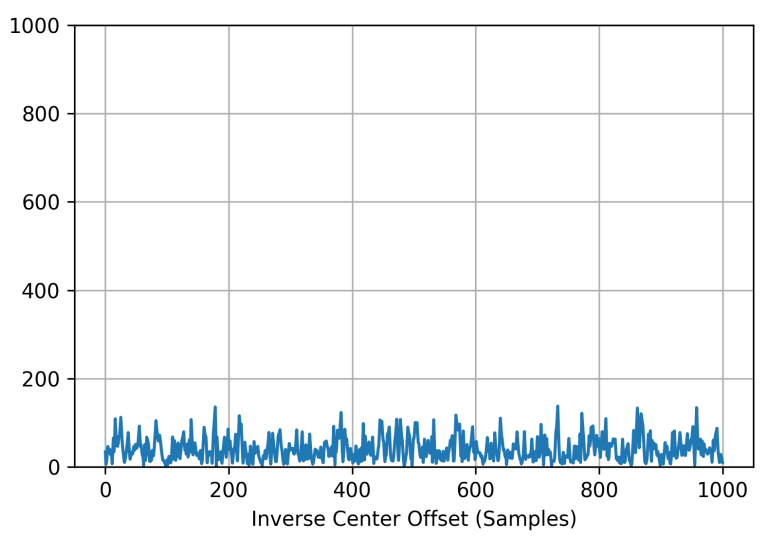

Fig. 15: Cross correlation of a frequency shifted signal.

corresponding cross correlations. A reference buffer and a capture buffer are instantiated in this module that provide the input to the pipeline as shown in Fig. 14. This module is a slave to a master as it is being driven by the data lines.

The results of a frequency shifted correlation is shown in Fig. 15, and an autocorrelation is shown in Fig. 16. We see that in Fig. 15 there is no peak. This is because two orthogonal signals should not have any correlation energy.

In the next code listing, the Python class definition for $C A F$

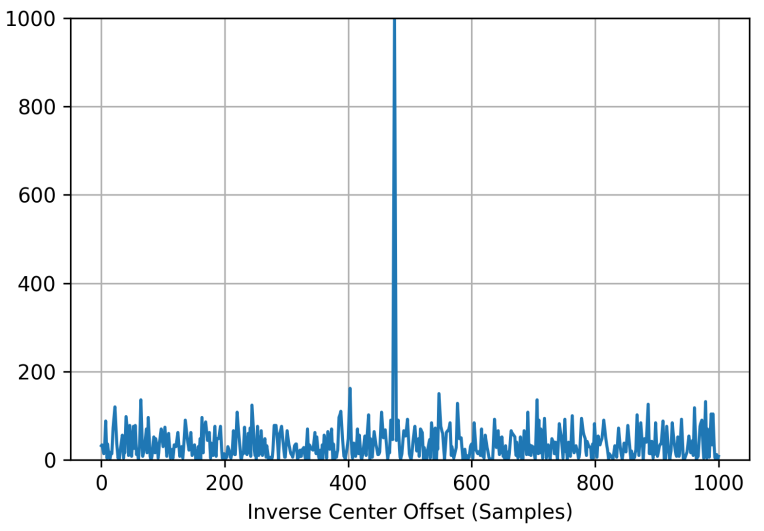

Fig. 16: Autocorrelation output with length of implemented design. 
TABLE 2: 12 phase bits, 8-bit multiplication, 49 frequencies, and 1000 samples.

$\begin{array}{llll}\text { Resource } & \text { Utilization } & \text { Available } & \text { Utilization \% } \\ \text { LUT } & 32682 & 53200 & 61.43 \\ \text { LUTRAM } & 490 & 17400 & 2.82 \\ \text { FF } & 28695 & 106400 & 26.97 \\ \text { BRAM } & 25.50 & 140 & 18.21 \\ \text { DSP } & 196 & 220 & 89.09 \\ \text { IO } & 53 & 125 & 42.40\end{array}$

is provided for reference. The class takes in both a reference and received or captured signal, and the number of bits requested to represent the signals. These two signals are required parameters. The reference signal is used to produce a stored reference as a capture buffer module, and the received signal is used in the generated test bench. The same parameters for the SigGen and FreqShift modules are required here as well, as they are passed down to their instantiations for the CAF to instantiate.

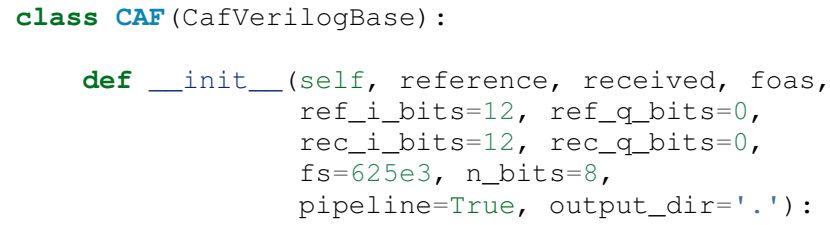

\section{Synthesis and Implementation}

Both the synthesis and implementation were completed successfully, and all timing constraints were met by the tool. Several different design sizes were elaborated and implemented, all ending up with different utilization amounts. The final design iteration that was able to maximize the iteration time is described by Table 2. Each of these tables describes a different usage that is still below the specific size of the Pynq board. For different devices, new CAF Python class instantiations should be used to explore board usages by using the Verilog module outputs to follow the Vivado design process.

The final implementation run shown by Table 2 was able to use the most of the resources of the board evenly because of the 8-bit multiplication [FWS]. The first couple implementations were using 12-bit numbers because that was what was nominally chosen for the simulations. However, since regenerating the module is very simple, a new CAF module was written out using the module and tested with different shifts. The final implementation has 49 different frequency offsets and an integration sample length of 1000 .

\section{Future Work and Enhancements}

When the original implementation of the sin and cosine generator was created, a half-sine method was used. While functionally sound, it is possible to decrease size by using a quarter-sine implementation where only a fourth of the sine is stored [Tec].

While the body of work for caf-verilog supports the modeling of the caf itself, this project can be used as a basis for incorporating Verilog as an extension to the wider scientific computing field. The SymPy Development Team has already made significant contributions in this realm, and is being used in many projects to support code generation for various languages such as $\mathrm{c}, \mathrm{c}++$, and Julia [Sym]. Using a common API, it should be possible to also provide an extension to incorporate the Verilog HDL.

\section{REFERENCES}

[ Na18] National Executive Committee for Space-Based Positioning, Navigation, and Timing . Gps.gov technical documentation, 2018. URL: https://www.gps.gov/technical/\#prn.

[Ana] Analog Devices. Ad7903 datasheet. URL: https://www.analog.com/ en/products/ad7903.html.

[Arm17] Arm Limited. Amba axi and ace protocol specification documentation, 2017. URL: https://developer.arm.com/docs/ihi0022/latest.

[FR] Ambrose Finnerty and Herve Ratigner. Reduce power and cost by converting from floating point to fixed point. URL: https://www.xilinx.com/support/documentation/white_papers/ wp491-floating-to-fixed-point.pdf.

[FWS] Yao Fu, Ephrem Wu, and Ashish Sirasao. 8-bit dot-product acceleration. URL: http://xilinx.com.

[Har05] Glenn D. Hartwell. Improved geo-spatial resolution using a modified approach to the complex ambiguity function (caf), 2005. URL: https: //calhoun.nps.edu/handle/10945/2033.

[HP17] John L. Hennessy and David A. Patterson. Computer Architecture, Sixth Edition: A Quantitative Approach. Morgan Kaufmann Publishers Inc., San Francisco, CA, USA, 6th edition, 2017.

[KPK81] W. C. Knight, R. G. Pridham, and S. M. Kay. Digital signal processing for sonar. Proceedings of the IEEE, 69(11):1451-1506, Nov 1981. doi:10.1109/PROC.1981.12186.

[Min13] Kwa Tak Ming. The Fundamental Operations in Bead Arithmetic How to Use the Chinese Abacus. France Press, 2013.

[MS] Eva Murphy and Colm Slattery. Ask the application engineer-33: All about direct digital synthesis. URL: https://www.analog.com/en/ analog-dialogue/articles/all-about-direct-digital-synthesis.html.

[Num] NumPy Developers. Numpy. URL: http://www.numpy.org/.

[Pro] Project Jupyter. Project jupyter. URL: https://jupyter.org.

[Ron] Armin Ronacher. Jinja 2 (the python template engine). URL: http: //jinja.pocoo.org/.

[Sida] Chiranth Siddappa. Caf verilog. URL: https://github.com/ chiranthsiddappa/caf_verilog.

[Sidb] Chiranth Siddappa. caf-verilog. URL: https://pypi.org/project/cafverilog/.

[Ste81] S. Stein. Algorithms for ambiguity function processing. IEEE Transactions on Acoustics, Speech, and Signal Processing, 29(3):588599, June 1981. doi:10.1109/TASSP.1981.1163621.

[Sym] SymPy Development Team. Codegen - sympy documentation. URL: https://docs.sympy.org/latest/modules/utilities/codegen.html.

[Tec] Gissequalt Technologies. Building a quarter sine-wave lookup table. URL: https://zipcpu.com/dsp/2017/08/26/quarterwave.html.

[Tum] Greg Tumbush. Signed arithmetic in verilog 2001 - opportunities and hazards. URL: http://www.tumbush.com/published_papers/.

[ver01] Ieee standard verilog hardware description language. IEEE Std 1364 2001, pages 1-792, Sep. 2001. doi:10.1109/IEEESTD.2001. 93352

[Wei94] L. G. Weiss. Wavelets and wideband correlation processing. IEEE Signal Processing Magazine, 11(1):13-32, Jan 1994. doi:10. $1109 / 79.252866$

[WSa] Mark Wickert and Chiranth Siddappa. Gps helper. URL: https://gpshelper.readthedocs.io/en/latest/?badge=latest.

[WSb] Mark Wickert and Chiranth Siddappa. Scikit dsp comm. URL: https://scikit-dsp-comm.readthedocs.io/en/latest/digitalcom.html.

[Xila] Xilinx Inc. Cordic v6.0. URL: https://www.xilinx.com/support/ documentation/ip_documentation/cordic/v6_0/pg105-cordic.pdf.

[Xilb] Xilinx Inc. Python productivity for zync (pynq). URL: https://pynq. readthedocs.io/en/v2.3/.

[Xilc] Xilinx Inc. Running the generate programming file process for fpgas. URL: https://www.xilinx.com/support/documentation/sw_ manuals/xilinx11/ise_p_generate_fpga_programming_file.htm.

[Xil14] Xilinx Inc. Vivado design suite user guide: Design flows overview, 2014. URL: https://www.xilinx.com/support/documentation/sw_ manuals/xilinx2014_1/ug892-vivado-design-flows-overview.pdf.

[ZT08] Rodger E. Ziemer and William H. Tranter. Principles of Communications. Wiley Publishing, 6th edition, 2008. 